\title{
SAR Imaging Features of Shallow Water Bathymetry
}

\author{
P. D. Pivaev 1, *, V. N. Kudryavtsev ${ }^{1,2}$, E. A. Balashova1, B. Chapron ${ }^{1,3}$ \\ ${ }^{1}$ Russian State Hydrometeorological University, Saint-Petersburg, Russian Federation \\ ${ }^{2}$ Marine Hydrophisical Institute of RAS, Sevastopol, Russian Federation \\ ${ }^{3}$ Institute Francais de Recherche pour I'Exploitation de la Mer, Plouzane, France \\ *pivaev.pavel@gmail.com
}

Purpose. The aim of the article is to study manifestations of the underwater topography features in the northern part of the White Sea in the images made by the spaceborn synthetic aperture radars (SAR) Sentinel-1A, Sentinel-1B.

Methods and Results. In the SAR images, the bottom features are revealed as bright and dark brightness anomalies. The anomalies were observed at the wind speed ranging from 2.6 to $10.8 \mathrm{~m} / \mathrm{s}$, and became reverse (bright anomalies turned dark and vice versa), when a tidal current changed its direction. It is shown that the observed SAR imagery contrasts correlate to divergence of a current formed by interaction of a tidal flow with the bottom topography inhomogeneities. The simulated SAR contrasts agree with the observations, and confirm the relation between the observed SAR contrasts and the current divergence. Together with modeling the SAR contrasts, the contribution of different mechanisms to formation of the observed modulations of the normalized radar cross section was qualitatively estimated. The wave breaking effect was especially accentuated. The method for retrieving the underwater bottom topography based on the relationship between the bottom gradient and the SAR imagery contrasts is proposed.

Conclusions. Location of the bottom inhomogeneities in the shallow water region on the whole coincides with that of the tidal current divergence and convergence zones, which are observed as brightness anomalies in the SAR images. Breaking of surface waves is the main contributor to the observed SAR contrasts. The bottom topography reconstructed from the SAR contrasts, and the actual one resulted from the bathymetry maps are in good agreement. Some discrepancies are interpreted as possible changes in depth and shape of the bottom topography features induced by action of strong currents and waves.

Keywords: bottom topography, Sentinel-1, SAR, wave breaking, current divergence.

Acknowledgments: the work was carried out within the framework of project No. 17-77-30019 supported by Russian Science Foundation; the authors are grateful to A.V. Zimin for providing the bottom topography data of the White Sea.

For citation: Pivaev, P.D., Kudryavtsev, V.N., Balashova, E.A. and Chapron, B., 2020. SAR Imaging Features of Shallow Water Bathymetry. Physical Oceanography, [e-journal] 27(3), pp. 290304. doi:10.22449/1573-160X-2020-3-290-304

DOI: $10.22449 / 1573-160 X-2020-3-290-304$

(C) P. D. Pivaev, V. N. Kudryavtsev, E. A. Balashova, B. Chapron, 2020

(C) Physical Oceanography, 2020

\section{Introduction}

Information on bottom topography is of utmost importance for safe shipping, pipe and cable rooting, planning and carrying out dredging operations. In some shallow areas (for example, the coast of Germany, the Netherlands, the Russian Arctic), fairways are laid in water areas with different bottom sediments. These sediments can be washed away and moved by intense currents or storms, which may result in filling and shallow water migration and pose a potential threat to shipping. 
It is well known that spaceborn synthetic aperture radars (SAR) are an effective tool for studying and monitoring various oceanic phenomena and processes, in particular, changing of the shape of underwater topography features (see chapter 10 in [1, pp. 245-262]). Under favorable conditions (moderate wind and strong current), bottom topography inhomogeneities can be manifested in the form of local contrasts of SAR images, which was described in a number of works [2-8].

One of the important practical applications of this effect is the inverse problem solution - underwater topography reconstruction based on the data of SAR measurements. Examples of solving this problem are presented, for example, in $[2,7-11]$. The inverse problem in $[9,10]$ is based on refraction effects that relate the kinematic characteristics of surface waves to the underwater topography in shallow water, and on the estimate of wave energy dissipation rate near the coast. In [11], the solution of the inverse problem was based on a formal mathematical apparatus that did not take into account the phenomenon nature.

There are also other approaches to solving this problem, when a connection is established between the contrasts of radar images with the characteristics of wind waves. Contrasts modulated by surface currents induced by the interaction of the main flow with underwater topography inhomogeneities, are of particular interest to us. In [2, 3, 7 and 8], the Bragg model is used as the basic radar scattering model. Correspondingly, the transformation of a short wind ripple (on the Bragg wave number) on the currents should lead to the formation of contrasts of the underwater topography SAR images. Using this approach to solving the inverse problem showed that the Bragg scattering model significantly underestimates the contrasts of SAR images. So, the usage of this model demands introduction a number of artificial assumptions that severely limit the practical application of the algorithms developed.

A significant underestimation of the model contrasts of the manifestation of various nature currents in $\mathrm{X}$ - and C-band SAR images, as compared with observations, was noted in previous works [5, 12]. As shown in [13] (see also $[14,15])$, the main reason for this discrepancy is the Bragg theory imperfection for describing the normalized radar cross section (NRCS) of the sea surface, radar reflections from breaking waves significantly contributing to backscatter and being the determining mechanism in the formation of the manifestation of currents in SAR images. At that, the anomalies of the breaking and the corresponding SAR signal contrasts are tied to the divergence zones of the currents.

The present article is aimed at establishing quantitative characteristics of the relationship between the SAR image contrasts, the underwater topography features and current parameters based on observational and simulation data.

Chapter 2 describes the area of observations and the initial data used in this work. Chapter 3 describes the processing of SAR images, as well as a joint analysis of SAR contrasts, divergence of the current and underwater topography. A simulation of the SAR contrasts with a contribution assessment of various backscatter formation mechanisms is given in Chapter 4. Chapter 5 demonstrates the underwater topography reconstruction using SAR data. 


\section{Study area and initial data}

The SAR manifestations of underwater topography in the northern White Sea - in the so-called Voronka (Funnel) of the White Sea are considered in the present work (Fig. 1). Stationary structures in the NRCS field of SAR images, presumably associated with the manifestations of bottom inhomogeneities, can be observed in all SAR images of this region.

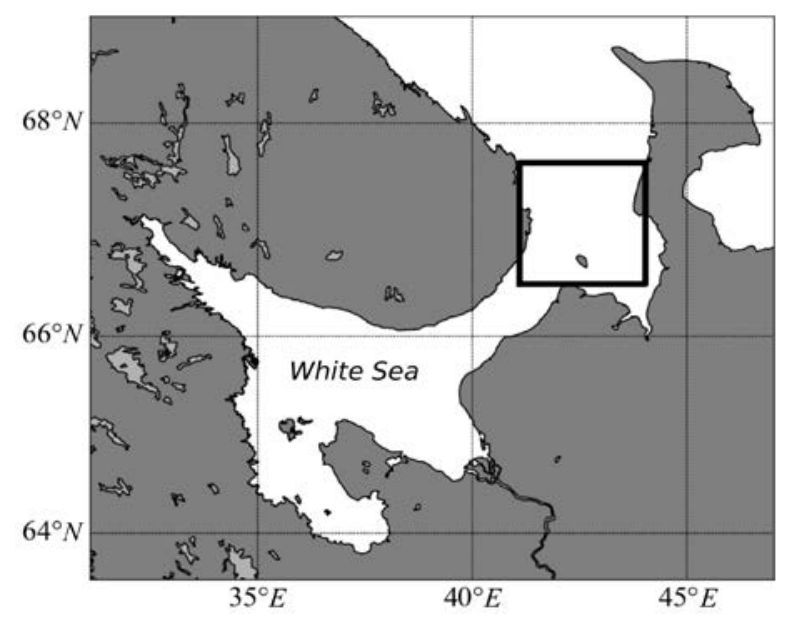

F i g. 1. Map of the White Sea. The region (called Voronka) investigated using the SAR images is marked out by a rectangle

This paper presents an analysis of 14 SAR images obtained from the Sentinel-1A and Sentinel-1B satellites of the European Space Agency (ESA) in Extra Wide Swath (EW) mode with HH-polarization in the summer-autumn period 2016 -2018. In the selected images, the spatial variations of the wind velocity field were minimal, there were no signal distortions caused by other phenomena (for example, internal waves and surface films), which permitted to analyze the underwater topography manifestations in their pure form. The SAR images used are $1^{\text {st }}$ level processing data of the Ground Range Detected (GRD) type. At the first stage of processing, thermal noise was removed from these data, then the images were transformed into the Arctic Projection (EPSG: 3413: NSIDC Sea Ice Polar Stereographic North/WGS84) with $40 \times 40 \mathrm{~m}$ resolution. The SAR image fragment example in the mentioned study area is shown in Fig. 2.

SAR data interpretation requires information on the depth, current velocity and near-water wind fields. The underwater topography fields were kindly provided by A.V. Zimin (St. Petersburg branch of Shirshov Institute of Oceanology of RAS). The depth in the study area does not exceed $70 \mathrm{~m}$ (Fig. 3). Shallow-water areas mainly run along the coasts of land and Morzhovets Island. They are also represented by alternating and oblong banks, the depth over which does not exceed $15-20 \mathrm{~m}$. 


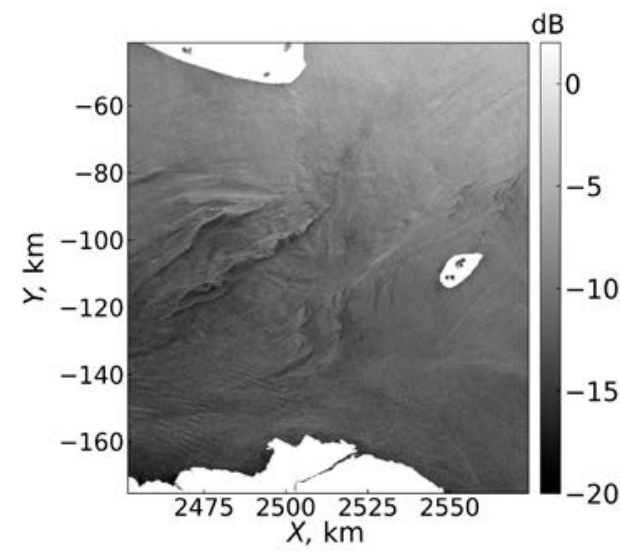

F i g. 2. Example of the SAR image of the White Sea Voronka (in the Arctic projection) obtained by Sentinel-1B on 25.07.2017 at 03:58 UTC

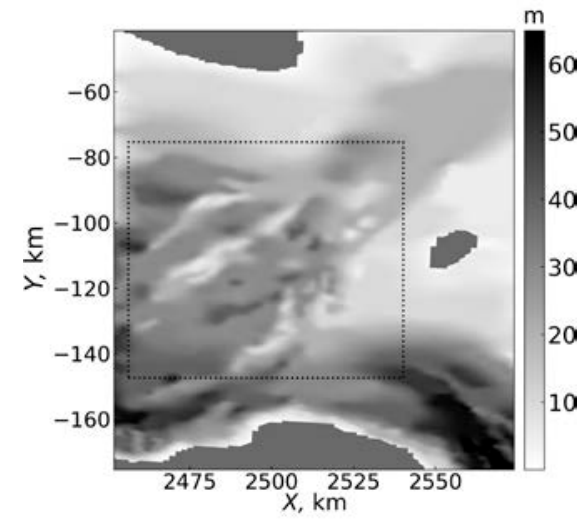

F i g. 3. Bottom topography map of the White Sea Voronka (location of the main banks under study is marked by a rectangle)

The tidal current velocity fields were taken from the White Sea Atlas of the Navy Hydrographic Service (1962) (URL: http://parusanarod.ru/bib/map/ws/tide/index.htm). The Atlas shows the speed and direction of currents at different points of the White Sea for a certain number of hours before the high tide on Sosnowets Island, located in the White Sea Throat. To obtain the current fields, corresponding to SAR-images acquisition time moments, the results of the tidal predictions at Sosnowets Island location were used. They are available on the website of the Unified State System of Information on the Situation in the World Ocean (URL: http://esimo.oceanography.ru/tides/). According to the Atlas, tidal currents in this area can be quite intense (the speed reaches $1.5-2 \mathrm{~m} / \mathrm{s}$ ) and alternating, due to their nature. Taking into account this specificity, it can be assumed that radar images were obtained under conditions of different phases of the local tide. The initial data on the sea depth and currents were then interpolated onto the Arctic projection grid with $40 \times 40 \mathrm{~m}$ resolution.

As data on the near-water wind speed, the ERA-Interim reanalysis results from the ECMWF archive (URL: https://www.ecmwf.int/), averaged over the area covered by the SAR image, were used.

\section{Relationship between SAR image contrasts and bottom topographycharacteristics}

As noted above, at first, thermal noise was removed from the original SAR images. Further, in order to suppress speckle noise, SAR images were smoothed using an additive Lee filter [16] with a window size of $10 \times 10$ pixels. To remove the NRCS dependence on the incidence angle, as well as on "large-scale" signal variations associated with the non-uniform wind field, a moving average filter with a window size of $400 \times 400$ pixels was applied to the SAR images. The images obtained in this way were presented in the form of contrast fields as:

$$
\sigma^{\prime}=\sigma /\langle\sigma\rangle-1,
$$


where $\sigma$ is the SAR contract field; $\sigma$ is the original despeckled SAR image; $\langle\sigma\rangle$ is the original despeckled image which a moving average filter was applied to.

Fig. 4 shows the average positive and negative contrasts in each of the images as a function of wind speed. When constructing it, only contrast values exceeding two standard deviations were taken into account. As follows from this figure, with the wind speed increase, the SAR signal contrasts decrease, but they are large enough to be identified in SAR images at wind speeds up to $11 \mathrm{~m} / \mathrm{s}$.

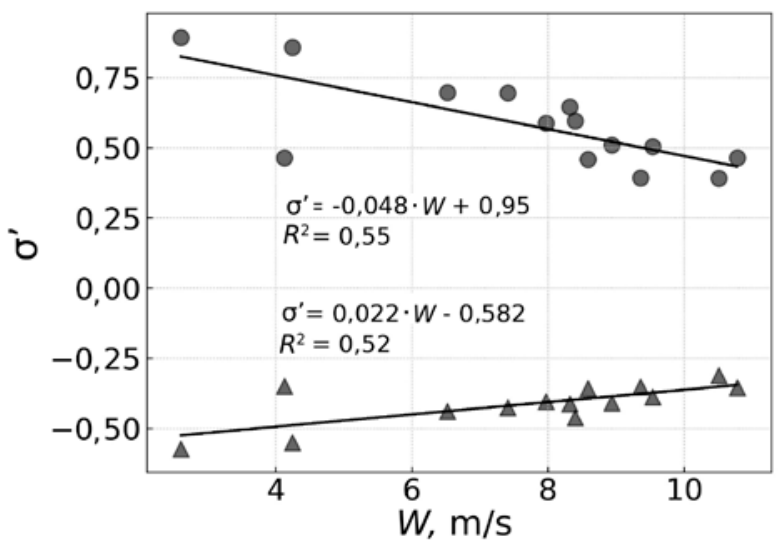

$\mathbf{F}$ i g. 4. Average values of positive $(\bullet)$ and negative $(\boldsymbol{\Delta})$ contrasts, exceeding two standard deviations of all the SAR images, as the function of wind speed

Along with the stationarity of the arrangement (i.e., the repeatability of the shape of the NRCS contrasts from image to image), an important feature was revealed in the fields of SAR contrasts, which was noted before in $[17,18]-$ in some SAR images, the contrast fields changed sign, i.e., became inverse. The sign change, as will be shown below, is associated with a change in the tidal flow direction (from the direction toward the White Sea to the opposite one).

For this reason, all the SAR data were divided into two groups: to the first one the NRCS fields and the corresponding fields of currents with one relative position of contrasts (11 scenes) were assigned, and to the second - with the opposite position (3 scenes). Further, the contrast fields of SAR images and currents were averaged within each of the groups. The contrast fields were averaged taking into account the variance:

$$
\left\langle\sigma^{\prime}\right\rangle=\frac{\sum_{i} \sigma_{i}^{\prime} \operatorname{std}\left(\sigma_{i}^{\prime}\right)}{\sum_{i} \operatorname{std}\left(\sigma_{i}^{\prime}\right)},
$$

where $\left\langle\sigma^{\prime}\right\rangle$ is the averaged contrast field of one data group; $\sigma_{i}^{\prime}-i$-field of NRCS contrasts in the group; $\operatorname{std}(\bullet)$ is the standard deviation operator.

The weighted averaging procedure also makes it possible to isolate a stationary spatially localized signal associated with non-uniform bottom topography manifestations and minimize NRCS variations caused by random variability of wind 
speed and manifestations of unsteady processes in the upper sea layer (for example, the passage of internal waves).
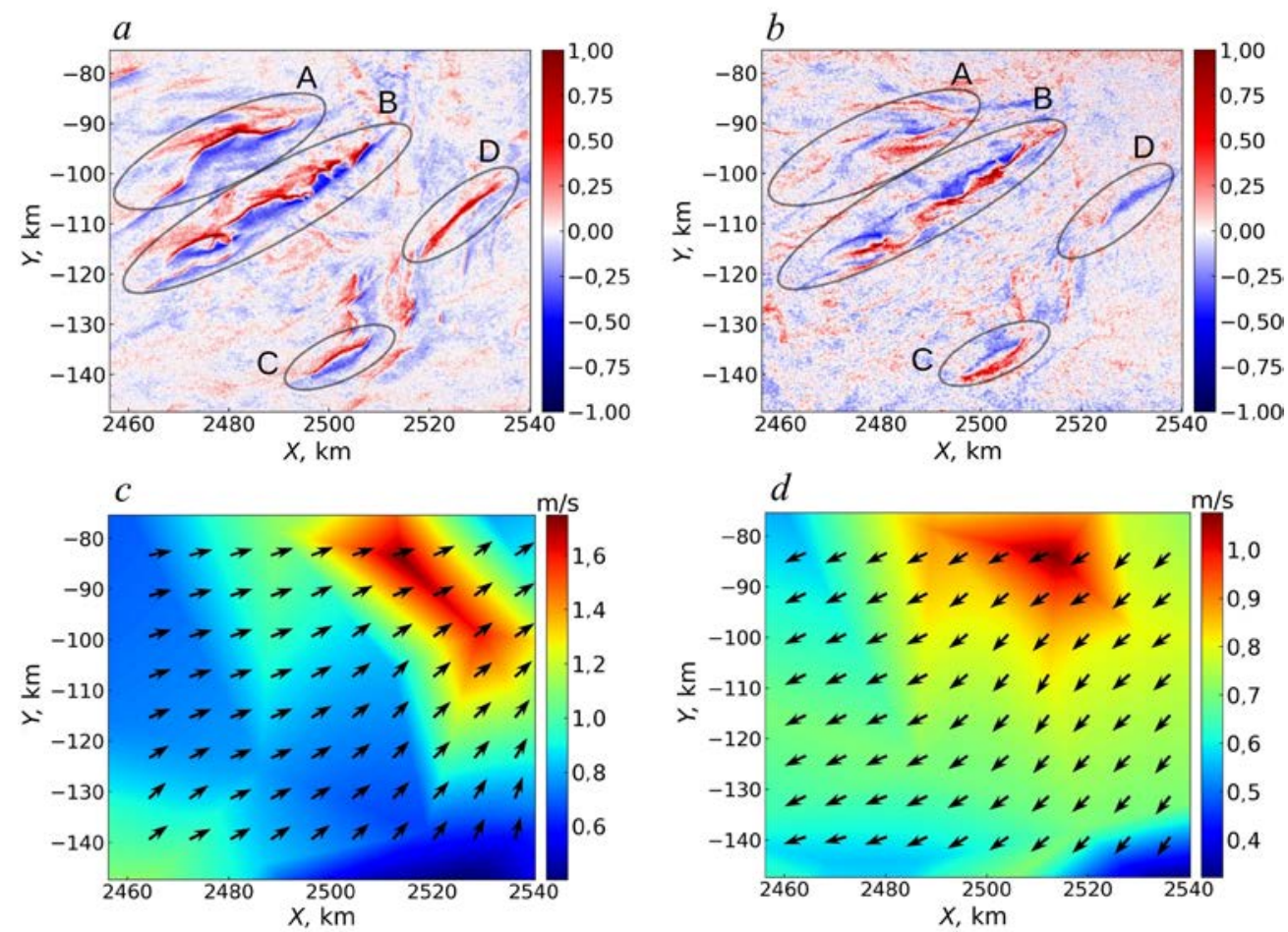

F i g. 5. Average SAR contrasts fields resulted from the first data group using 11 images $(a)$ and from the second data group using 3 images $(b)$; tidal current fields for the first data group $(c)$ and for the second data group $(d)$

The contrast fields of the SAR images and tidal currents for each of the groups are presented in Fig. 5. As can be seen from the average fields of the currents with generally opposite directions shown on Fig. 5, $c$ and Fig. 5, $d$, for both data groups the assumption of a sign change of the SAR contrasts along with a change in the direction of the tidal current is confirmed. Four pronounced manifestations of the bottom topography inhomogeneities (indicated by ellipses in Fig. 5, $a, 5, b$ ) can be distinguished in the fields of NRCS contrasts: B and C features are manifested with almost the same intensity in different phases of the tide. However, A and D features in Fig. 5, $b$ do not have the same pronounced manifestation as in Fig. 5, $a$, which can be explained by unstable signal variations at a given place in the corresponding group of images and by relatively small amount of the images comprising this group.

Following the work [13], it is assumed that the SAR signal anomalies, caused by the currents, are related to the divergence field of the latter: the SAR signal is amplified in the zones of convergence of currents and weakened in their divergence zones. Since the scale of generation of Bragg waves (of the order of $10 \mathrm{~m}$ ) is much smaller than the scale of the variability of currents in the study area, the observed 
features may be due to the wave breaking modulation. The wave breaking, having a noticeable (but not determinative) contribution to backscatter, is highly sensitive to the gradients of currents and intensifies in convergence zones of currents and weakens in their divergence zones. Reasoning from this fact, the bottom topography manifestations in SAR images can be interpreted as manifestations of the convergence/divergence zones of the currents caused by the interaction of the main tidal current with the non-uniform bottom topography.

From the shallow water theory it follows that the current velocity divergence $u$ is associated with a local depth gradient $H$ by the following relation:

$$
\nabla \cdot \boldsymbol{u}=-(\boldsymbol{u} \cdot \nabla H) / H .
$$

As follows from Fig. 3 and 5, bottom inhomogeneties are elongated along the direction of the tidal current. For this reason, to interpret the SAR-data observations as a first approximation, we will consider the one-dimensional expression (1), where locally the $\mathrm{X}$ axis is directed along the main orientation of the SAR contrasts (perpendicular to the steepest descent line), and the $\mathrm{Y}$ axis in perpendicular to the $\mathrm{X}$ axis direction. Assuming a small depth gradient along the $\mathrm{X}$ axis and, consequently, the absence of divergence in this direction, expression (1) takes the form as follows

$$
\partial v / \partial y=-\left(v_{0} H_{0} / H^{2}\right) \partial H / \partial y,
$$

where $v$ is the velocity component in the $\mathrm{Y}$ axis direction; $v_{0}$ and $H_{0}$ are the background values of velocity and depth (beyond a bottom non-uniformity). From relation (2) it follows that the greater the sea depth is, the lower the induced current velocity and divergence are. Since the SAR contrasts are determined by the divergence of the currents, it can be expected that the bottom topography manifestations in SAR images in the shallow sea will be more pronounced and disappear in deep water.

Comparison of the SAR contrasts with the divergence of the current around a given depth profile was carried out along four transections drawn through the distinguished features of the contrast field in Fig. 5. Profiles of SAR contrasts, sea depths and divergences are shown in Fig. 6, where a moving average filter with a window size equal to 10 units of discreteness was applied to the transections to minimize the residual random NRCS fluctuations. To calculate the divergence, the projection of the average field of the current velocity onto the direction of each of the transections was used. 

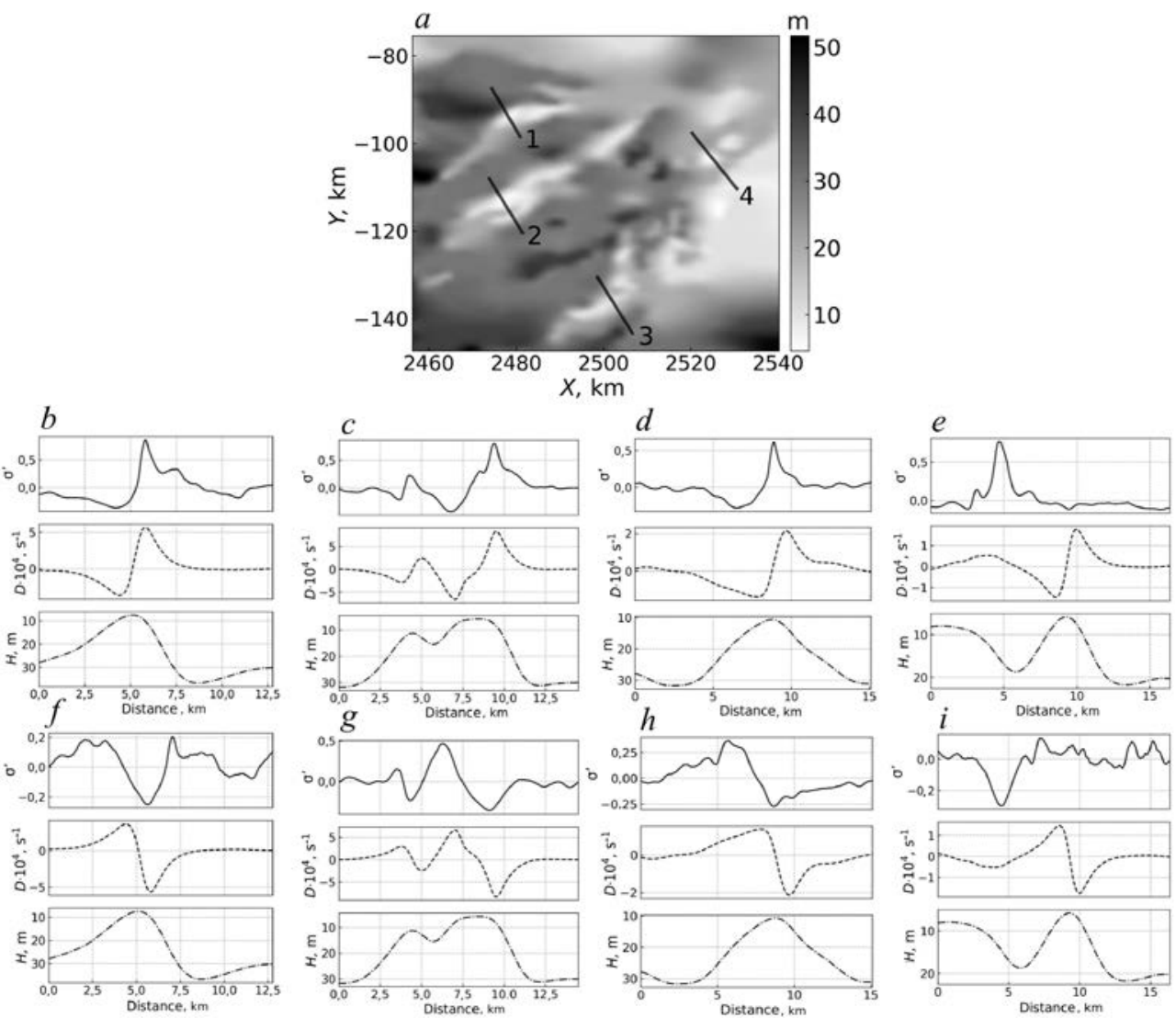

F i g. 6. Bottom topography of the marked area of SAR images $(a)$, the lines with numbers denote the transections $1(b), 2(c), 3(d)$ and $4(e)$ of the SAR contrasts (solid line), current divergence with the negative sign (dashed line) and bottom relief (dash-dotted line) when a tidal current is directed to the sea; the same sections but the tidal current direction is reverse $(f, g, h, i)$

As seen from Fig.6, the SAR signal contrasts are localized at relatively large divergence/convergence values, which, in turn, are located on the slopes of the banks. Positive SAR contrasts coincide with the convergence zones, and negative ones - with the divergence zones of the current. Such consistency, as well as its conservation during a tidal phase variation, are clearly visible in the first three sections, Fig. $6, b-d$ and $f-h$. Note that in some cases there is a shift between the SAR contrasts and the divergence/convergence zones, which may be due to the difference between the actual (real) position of the slopes and the following from the bottom topography map in Fig. 3. An obvious discrepancy between SAR contrasts and divergence is observed in section 4 (Fig. 6, $e, 6, i$ ), which suggests that the actual difference in depths can be much larger than that on the map of the available bottom topography (Fig. 3).

\section{Radar signal simulation}

Below there is the interpretation of the SAR observation data based on the model for the formation of SAR images of the sea surface (hereinafter, the RIM model) 
proposed in [13]. In accordance with this model, the sea surface NRCS $\sigma_{0}^{p p}$ ( $p p$ denotes the polarization, for example, $v v$ or $h h$ ) at moderate and large incidence angles (over $25^{\circ}$ ) is represented as the sum of the resonant Bragg scattering (described in the two-scale model framework) $\sigma_{0 B}^{p p}$ and the radar reflections from breaking waves $\sigma_{w b}$ :

$$
\sigma_{0}^{p p}=\sigma_{0 B}^{p p}+\sigma_{w b} .
$$

The relative contribution of wave breaking to the NRCS, calculated by the formula $R_{w b}^{p p}=\sigma_{w b} / \sigma_{0}^{p p}$, is significant: on VV-polarization it varies within $30-50 \%$ on average, and on the HH-polarization it is about 50 to $65 \%$ of the total NRCS (see Fig. 2 in [14, pp. 3]) at a signal incidence angle of about $35.5^{\circ}$, which is specific to the center of fragments of the used SAR images. Relative contribution of wave breaking $R_{w b}^{p p}$ also strongly depends on the direction between the radar and the wind: it takes maximum values when the radar look direction is in crosswind, minimal - when it is downwind and intermediate - when it is upwind.

As shown in the RIM model, the manifestation of currents in SAR images is primarily due to the modulation of wave breaking. Due to the small relaxation scale (of the order of $10 \mathrm{~m}$ ), the spectra of C-band Bragg waves (wavelength $5.5 \mathrm{~cm}$ ) are not modulated by currents with a variability scale of the order of hundreds of meters or more, and their level is determined by the local wind speed. The contribution of wave breaking to the NRCS is proportional to the sea surface fraction covered by the breaking waves, which is formed in a wide spectral range of wavelengths - from a meter to wavelengths of the spectral peak. Accordingly, the wave spectrum in this range is sensitive to the effects of currents, which leads to a significant modulation of wave breaking, the intensity of which is a strongly non-linear function of the spectrum level.

Fig. 7 illustrates the formation features of the bottom topography manifestation in SAR images. Transection 1 (see Fig. 6) was chosen as an example for the case when the current velocity vector is pointing to the White Sea.

Fig. 7, $a, 7, c$, and 7, $e$ show the dependence of the sea surface NRCS contrasts on the angle between the near-water wind and radar look direction, as well as the contribution of various scattering mechanisms - the Bragg scattering and radar reflections from breaking waves - to the formation of SAR contrasts. Note that the modulations of the spectrum of the Bragg ripple by the currents are insignificant, which is due to the small relaxation scale of the Bragg waves (about $10 \mathrm{~m}$ ), compared with the spatial variability of the currents (about $1 \mathrm{~km}$ ). Correspondingly, SAR models based only on the Bragg scattering cannot explain the observed contrasts of SAR images. At first glance, this conclusion contradicts the results of [2, 3 and 7], however, the Bragg model there was in qualitative agreement with the observational data as a result of adjustment or parameterization of the ripple relaxation scale to reproduce the observed SAR contrasts. Unlike the indicated works, the relaxation scale in the RIM model is not a free parameter, but has a fixed definition, which follows from the obvious fact that the spectrum reactions to disturbances caused by wind and current are physically equivalent (see section 3.1-3.2 in [13, pp. 9-11]). 

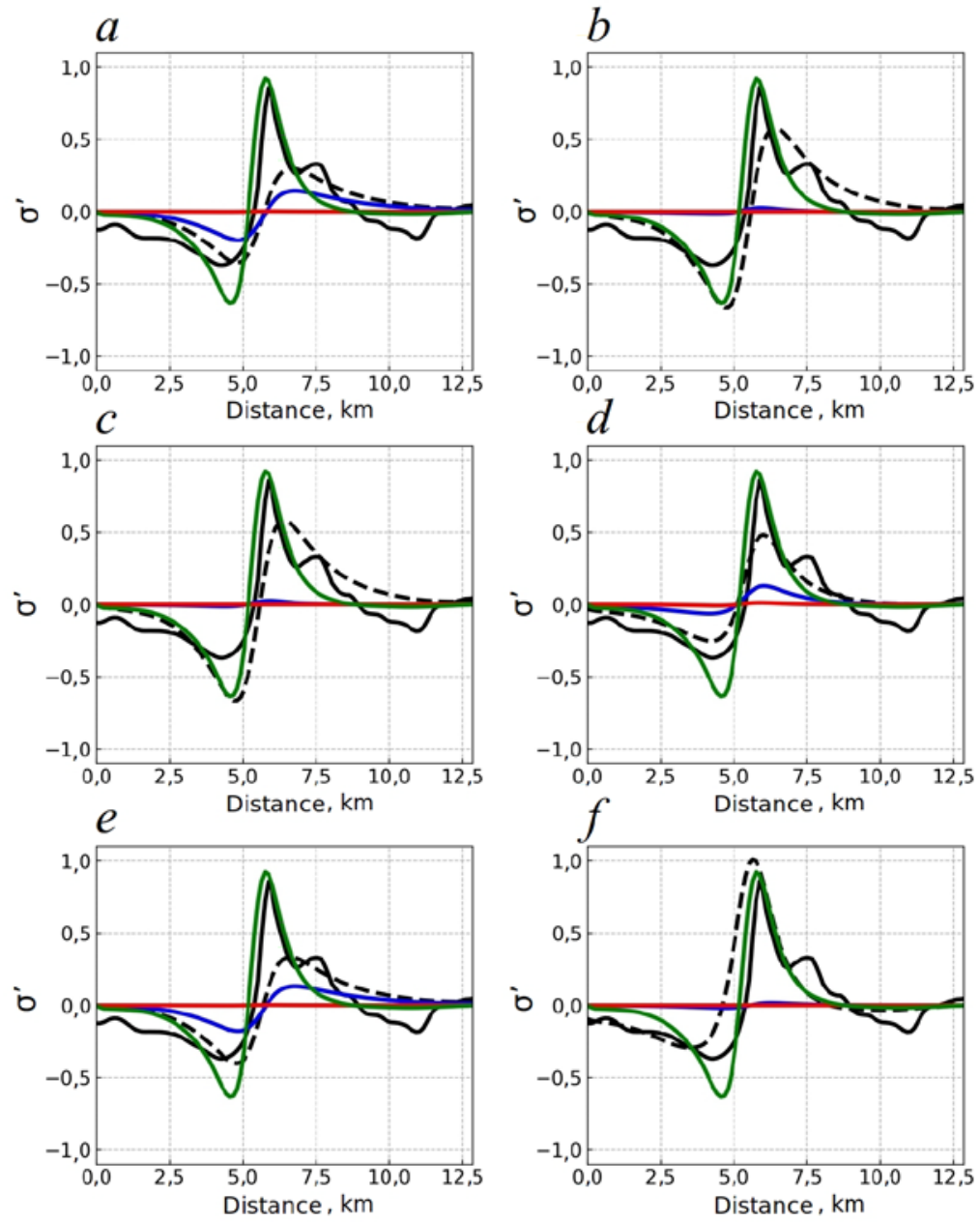

- Sentinel-1 $\left.(\sigma) \quad--\operatorname{RIM1}\left(\sigma^{\prime}\right)+\sigma^{\prime} \prime_{M S S}+\sigma_{\prime m o}\right)$

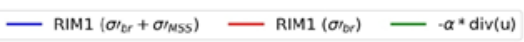

F i g. 7. Simulated normalized radar cross section contrasts for transection 1 (see Fig. 6) at the tidal current directed to the sea (projection of the current induced by the bottom inhomogeneity is positive in the transection direction) at the $7.5 \mathrm{~m} / \mathrm{s}$ wind speed: the wind vector is parallel to the current ( $a, c$, $e$ ) and the radar look is directed downwind $(a)$, crosswind $(c)$ and up-wind $(e)$; the radar look is crosswind $(b, d, f)$ and the wind vector is directed down-current $(b)$, crosscurrent $(d)$ and up-current $(f)$. Black solid line denotes the Sentinel-1 observed contrasts, black dashed line - the RIM contrasts, blue line - the two-scale Bragg scattering model contrasts and red line - the pure Bragg scattering contrasts

Unlike the Bragg model, the contrasts of the NRCS within the two-scale Bragg model become noticeable. In this case, the NRCS variations are caused by the variability of the mean squared slope (MSS) of long waves (with wavelengths several times longer than the length of the radio wave) carrying Bragg ripple. The relaxation scales of the low-frequency part of the spectrum of waves that form the MSS are comparable with the scales of currents. Therefore, in the two-scale Bragg model framework, the MSS variations over the current cause NRCS variations 
(the first term in expression (3)). The contribution of resonant scattering to the full NRCS variations is determined by the relative contribution of $\sigma_{0 B}^{h h}$ to the full NRCS $\sigma_{0}^{\text {hh }}$, which is minimal when the radar look direction is in crosswind position. As follows from Fig. 7, $c$, under these conditions, the contribution of resonant scattering to the formation of SAR contrasts is insignificant. Taking into account the contribution of wave breaking to the NRCS ( $2^{\text {nd }}$ term in expression (3)) leads to significant signal variations comparable with those observed.

Profile of the NRCS variations generally corresponds to the divergence profile of currents: an increase in the NRCS anomalies occurs in convergence zones and attenuation - in divergence zones. As shown in [13], among all the possible combinations of the components of the current vector gradient tensor, the divergence has a decisive influence on the modulation of the integral parameters of surface waves (such as, for example, the surface fraction covered by breaking waves or MSS). This explains the similarity of the profiles of NRCS variations (both the full and the two-scale Bragg NRCS) to the divergence profiles of currents induced by bottom topography. Fig. 7, $c$ also shows that the greatest NRCS contrasts should be expected when the radar looks in the crosswind direction, when the contribution of wave breaking to the sea surface NRCS is maximum, and the strongly nonlinear dependence of the wave breaking intensity on the spectrum level leads to significant radar contrasts.

Fig. $7, b, 7, d$ and $7, f$ illustrate the dependence of SAR contrasts on the direction between the wind vector and the current vector at a fixed angle between the radar and wind (in this case $90^{\circ}$ ) direction. The NRCS anomalies are maximum in the direction of the wind, i.e., in the direction of wave propagation towards the current. For all wind directions relative to the current, the spatial variations of NRCS approximately correspond to the divergence current profile with already noted signal amplification in the convergence zones and attenuation in the divergence zones. A certain shift of the NRCS maxima relative to the convergence maxima and its minima relative to the divergence maxima with variations in wind direction is noted.

However, it can be expected that, when averaging the SAR contrasts obtained for different wind directions, the general position of the anomalies of SAR images will be tied to the divergence zones of currents. It can be seen from the experimental data in Fig. 8, where the wind vector and radar direction are given as average values within the corresponding data group. It is also noted that, as in previous calculations, the dominant contribution to the formation of SAR contrasts is made by wave breaking modulations; the role of Bragg ripple spectrum modulations is insignificant, while the resonant scattering contribution in the framework of the two-scale model (due to MSS modulations) can be significant. 


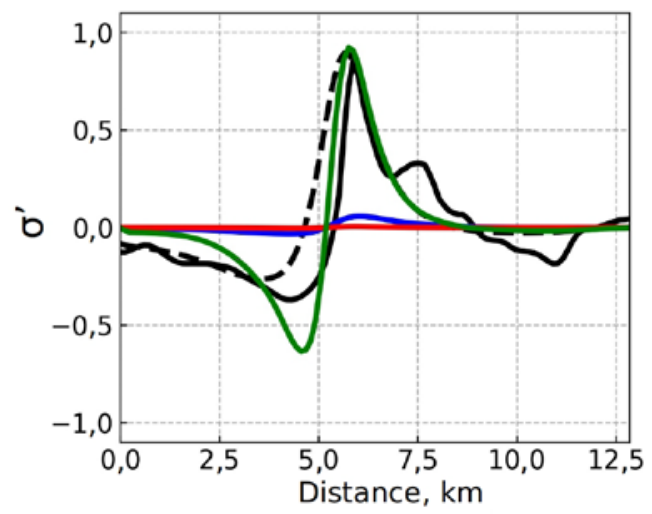

F i g. 8. Simulated normalized radar cross section contrasts for the same transection and at the same current as in Fig. 7. The notations are the same as in Fig. 7

\section{Depth estimation according to the SAR contrasts}

Qualitative correspondence of the SAR contrasts of the current divergence over the bottom inhomogeneities given a priori (see Fig. 8) allows considering the inverse problem - the basin depth estimation from the detected anomalies of the SAR signal. For this purpose the SAR contrasts $K$ are assumed to be proportional to the divergence of the surface currents: $K \propto \operatorname{div}(u)$, where the proportionality coefficient is a certain transfer function. As follows from the simulation results (see Fig. 7), this function may depend on the wind vector direction relative to the current vector and the radar direction, on the wind speed (see Fig. 4), and probably, on a number of other factors.

For simplicity, the bathymetry features are believed to be quasi-onedimensional. Then, using the linearized equation (2), the relationship of SAR contrasts and depth variations in the direction of the largest slope can be written in the following form:

$$
K=T \partial H / \partial y,
$$

where $T$ is the transfer function determining the depth reconstruction accuracy. In the present paper, the transfer function is believed to be unknown, however, the difference in depths in the considered region is known, which can be used to determine the unknown constant $T$ (calibration of the SAR contrasts). In this case, the goal is not to retrieve the absolute depth values, but the bottom topography shape, which may change under the influence of intense currents and storms or other natural or anthropogenic factors, for example.

To determine the actual shape of the bottom topography, the equation (4) with given depths at the two boundaries of the integration region is used. In this case, the problem is overdetermined, and an extra boundary condition is used to obtain the transfer function. 

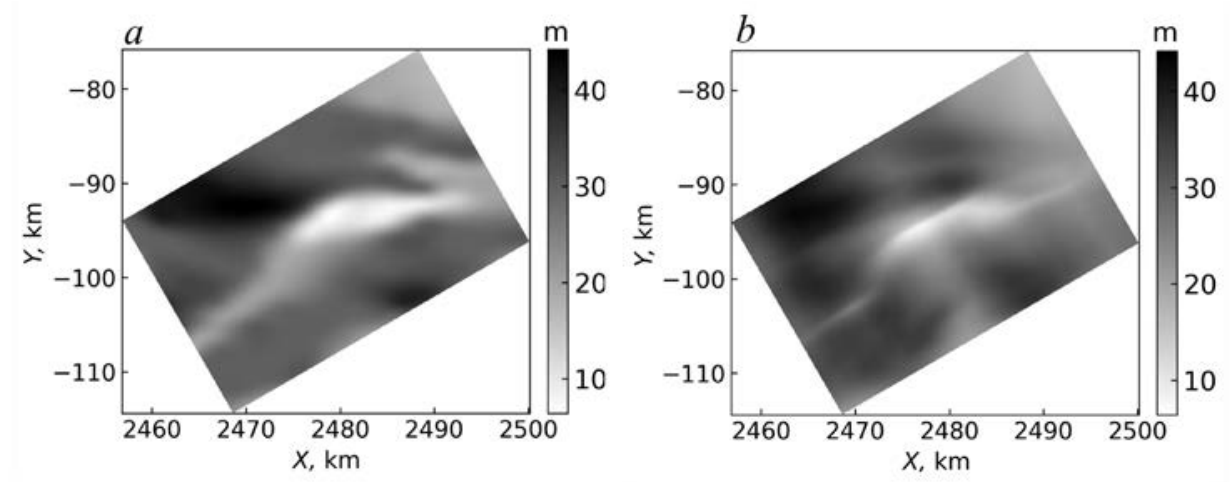

F i g. 9. Feature $A$ (see Fig. 5) of the bottom topography field (a); depth in the same region reconstructed by integration (4) (b)

Fig. 9, $b$ shows the reconstructed bottom topography feature $A$ (Fig. 9, $a$ ), which is manifested in the field of the SAR image contrasts. As the boundary conditions for solving the equation (4), the depths at the selected region boundary are used. The transfer function was set as a constant value for the entire region, and was determined from the condition of the minimum standard deviation of the reconstructed depth field from the given a priori. As expected, the shape of the retrived bank corresponds to that a priori given. The most important are the differences in the shape and position of the topographic elements of the reconstructed depth field and the one shown in Fig. 3. In particular, the depression slightly to the left of the main central elevation is displaced upward and more elongated; additional depth variations appeared in the lower part of the region; at the top right, the topography becomes more uniform, and the bank itself is narrower than the depth field in Fig. 9, $a$. Such differences can be interpreted as a modified configuration of the bottom topography, documented according to the SAR measurement data.

\section{Conclusion}

The specific features of the northern White Sea bottom topography are clearly manifested in the SAR images obtained from the Sentinel-1A and Sentinel-1B satellites. The bottom topography manifestations are the result of the interaction of wind waves with spatially inhomogeneous surface currents induced by the tidal current interaction with the non-uniform bottom topography.

It is shown that NRCS anomalies of images are associated with divergence of induced currents, the SAR signal amplification and attenuation taking place in the areas of convergence and divergence of currents, respectively.

The main mechanism for the formation of NRCS contrasts is the radar signal scattering on the breaking of wind waves. Sensitivity of these waves to inhomogeneities of surface currents is manifested in the form of the breaking intensity amplification and weakening, which takes place in the convergence and divergence zones, respectively. The role of resonant Bragg scattering in the formation of the SAR manifestations of bottom topography is insignificant. 
The possibility of bottom topography reconstruction from an averaged field of SAR contrasts was demonstrated. The basic bathymetry elements are quite well restored, the differences in the bottom location and shape, as well as local elevations, can be interpreted as actual changes in the bottom topography that have occurred from the time of the last in situ measurements until the time of the SAR observations considered in the present study.

\section{REFERENCES}

1. Jackson, C.R. and Apel, J.R., eds., 2004. Synthetic Aperture Radar Marine User's Manual. Washington, DC: U.S. Department of Commerce, 464 p.

2. Alpers, W. and Hennings, I., 1984. A Theory of the Imaging Mechanism of Underwater Bottom Topography by Real and Synthetic Aperture Radar. Journal of Geophysical Research: Oceans, 89(C6), pp. 10529-10546. https://doi.org/10.1029/JC089iC06p10529

3. Shuchman, R.A., Lyzenga, D.R. and Meadows, G.A., 1985. Synthetic Aperture Radar Imaging of Ocean-Bottom Topography via Tidal-Current Interactions: Theory and Observations. International Journal of Remote Sensing, 6(7), pp. 1179-1200. doi:10.1080/01431168508948271

4. Lodge, D.W.S., 1983. Surface Expressions of Bathymetry on Seasat Synthetic Aperture Radar Images. International Journal of Remote Sensing, 4(3), pp. 639-653. https://doi.org/10.1080/01431168308948580

5. Romeiser, R. and Alpers, W., 1997. An Improved Composite Surface Model for the Radar Backscattering Cross Section of the Ocean Surface: 2. Model Response to Surface Roughness Variations and the Radar Imaging of Underwater Bottom Topography. Journal of Geophysical Research: Oceans, 102(C11), pp. 25251-25267. https://doi.org/10.1029/97JC00191

6. Zheng, Q., Li, L., Guo, X., Ge, Y., Zhu, D. and Li, C., 2006. SAR Imaging and Hydrodynamic Analysis of Ocean Bottom Topographic Waves. Journal of Geophysical Research: Oceans, 111(C9), C09028. doi:10.1029/2006JC003586

7. Calkoen, C.J., Hesselmans, G.H.F.M., Wensink, G.J. and Vogelzang, J., 2001. The Bathymetry Assessment System: Efficient Depth Mapping in Shallow Seas Using Radar Images. International Journal of Remote Sensing, 22(15), pp. 2973-2998. https://doi.org/10.1080/01431160116928

8. Stewart, C., Renga, A., Gaffney, V. and Schiavon, G., 2016. Sentinel-1 Bathymetry for North Sea Palaeolandscape Analysis. International Journal of Remote Sensing, 37(3), pp. 471-491. doi:10.1080/01431161.2015.1129563

9. $\quad$ Brusch, S., Held, P., Lehner, S., Rosenthal, W. and Pleskachevsky, A., 2011. Underwater Bottom Topography in Coastal Areas from TerraSAR-X Data. International Journal of Remote Sensing, 32(16), pp. 4527-4543. doi:10.1080/01431161.2010.489063

10. Wackerman, C., Lyzenga, D., Ericson, E. and Walker, D., 1998. Estimating Near-Shore Bathymetry Using SAR. In: IEEE, 1998. IGARSS '98. Sensing and Managing the Environment. 1998 IEEE International Geoscience and Remote Sensing: Symposium Proceedings. Seattle, WA, USA: IEEE. Vol. 3, pp. 1668-1670. doi:10.1109/IGARSS.1998.692407

11. Inglada, J. and Garello, R., 2002. On Rewriting the Imaging Mechanism of Underwater Bottom Topography by Synthetic Aperture Radar as a Volterra Series Expansion. IEEE Journal of Oceanic Engineering, 27(3), pp. 665-674. doi:10.1109/JOE.2002.1040949

12. Caponi, E.A., Crawford, D.R., Yuen, H.C. and Saffman, P.G., 1988. Modulation of Radar Backscatter from the Ocean by a Variable Surface Current. Journal of Geophysical Research: Oceans, 93(C10), pp. 12249-12263. doi:10.1029/JC093iC10p12249

13. Kudryavtsev, V., Akimov, D., Johannessen, J. and Chapron, B., 2005. On Radar Imaging of Current Features: 1. Model and Comparison with Observations. Journal of Geophysical Research: Oceans, 110(C7), C07016. doi:10.1029/2004JC002505 
14. Kudryavtsev, V.N., Fan, S., Zhang, B., Mouche, A.A. and Chapron, B., 2019. On QuadPolarized SAR Measurements of the Ocean Surface. IEEE Transactions on Geoscience and Remote Sensing, 57(11), pp. 8362-8370. doi:10.1109/TGRS.2019.2920750

15. Fan, S., Kudryavtsev, V., Zhang, B., Perrie, W., Chapron, B., Mouche, A., 2019. On C-Band Quad-Polarized Synthetic Aperture Radar Properties of Ocean Surface Currents. Remote Sensing, 11(19), 2321. https://doi.org/10.3390/rs11192321

16. Lee, J.-S., Grunes, M.R. and Mango, S.A., 1991. Speckle Reduction in Multipolarization, Multifrequency SAR Imagery. IEEE Transactions on Geoscience and Remote Sensing, 29(4), pp. 535-544. doi:10.1109/36.135815

17. De Loor, G.P., 1978. Remote Sensing of the Sea by Radar. Analysis of Available Data and Results of Noordwijk '77. Hague, Netherlands: Physics LAB RVO-TNO, 59 p.

18. De Loor, G.P., 1981. The Observation of Tidal Patterns, Currents and Bathymetry with SLAR Imagery of the Sea. IEEE Journal of Oceanic Engineering, 6(4), pp. 124-129. doi:10.1109/JOE.1981.1145501

About the authors:

Pavel D. Pivaev, Technician of the Satellite Oceanography Laboratory, Russian State Hydrometeorological University (79, Voronezhskaya St., St. Petersburg, 192007, Russian Federation),pivaev.pavel@gmail.com

Vladimir N. Kudryavtsev, Head of the Satellite Oceanography Laboratory, Russian State Hydrometeorological University (79, Voronezhskaya St., St. Petersburg, 192007, Russian Federation), Dr. Sci. (Maths \& Physics), Leading Research Associate, Marine Hydrophysical Institute of RAS (2, Kapitanskaya St., Sevastopol, 299011, Russian Federation); Scopus Author ID: 7102703183,kudr@rshu.ru

Ekaterina A. Balashova, Programmer Division Leader of the Satellite Oceanography Laboratory, Russian State Hydrometeorological University (79, Voronezhskaya St., St. Petersburg, 192007, Russian Federation), balashova@rshu.ru

Bertrand Chapron, Scientific Supervisor the Satellite Oceanography Laboratory, Russian State Hydrometeorological University (79, Voronezhskaya St., St. Petersburg, 192007, Russian Federation), Senior Research Associate of the Satellite Oceanography Laboratory, National Institute for Ocean Science (Institute Francais de Recherche pour I'Exploitation de la Mer) (Centre Bretagne, ZI de la Pointe du Diable, CS 10070, 29280 Plouzané), Scopus Author ID: 56209544000, bertrand.chapron@ifremer.fr

Contribution of the co-authors:

Pavel D. Pivaev - review and analysis of literature on the problem, data collection, software development, data processing and analysis and writing the paper text

Vladimir N. Kudryavtsev - problem statement, data analysis and the paper editing

Ekaterina A. Balashova - collection of SAR images and their preliminary processing

Bertrand Chapron - problem statement, discussion of the results and the project financial support

The authors have read and approved the final manuscript.

The authors declare that they have no conflict of interest. 